\title{
EFFECT OF SCREENING ON RESISTIVITY OF LIQUID METALS
}

\author{
K.A. AZEZ AND I.A. Al-OMari \\ Department of Physics, Faculty of Science \\ Jordan University of Science and Technology, Irbid, Jordan \\ (Received May 22, 1996; revised version February 28, 1997; \\ in final form May 6, 1997)
}

\begin{abstract}
Calculations of the resistivity of liquid metals are performed basing on the structure factor $a(q)$ which can be measured experimentally or calculated theoretically from the hard sphere model. The calculations are carried out by using the Ashcroft empty core pseudopotential in the framework of Ziman's formula along with different forms of the screening function due to Ilartree, Geldart and Vosko, IIubbard, Overhauser, random phase approximation and self-consistent screening. The investigation is useful because it throws light on the importance of exchange and correlation effects in developing an appropriate description of the screening of ions by conduction electrons for calculating the resistivity of liquid metals.
\end{abstract}

PACS numbers: $72.15 . \mathrm{Cz}$

\section{Introduction}

In recent years considerable efforts have been made (March [1] and Young [2]) to describe the properties of liquid metals by blending the techniques of classical liquid state theory with that of the pseudopotential method. This was first used successfully by Stroud and Ashcroft [3] to describe the melting of $\mathrm{Na}$. After this initial success considerable interest was generated among other workers who studied various. properties of liquid metals and alloys (Umar et al. [4], Hafner [5], Singh [6], Regnault [7] and Pandey et al. [8]).

The major task in such a study is to choose an appropriate reference system among the various existing methods namely:

(i) IIard sphere and variational method (Jones [9]),

(ii) IIard sphere and interionic pair potential (Ashcroft and Langreth [10]),

(iii) Weeks-Chandler-Andersen method (Weeks et al. [11]),

(iv) One-component plasma system (ITansen [12]). 
Among the numerous existing methods, the use of a hard sphere model is largely justified because it provides an analytical representation in terms of a single parameter known as the hard sphere diameter $(\sigma)$. Previous investigations (Azez et al. [13-15]) are very useful for describing the electron-ion behaviour of liquid metals. In the present paper, we attempt to go further in this direction, while investigating the effect of ionic screcning by the conduction electrons. The electrical resistivity of liquid metals $\mathrm{K}, \mathrm{Al}, \mathrm{Sn}$ and $\mathrm{Bi}$ are calculated with different forms of the screening functions. Our study suggests that a hard sphere system serves as a good model for these liquid metals near the melting temperatures.

\section{Theory}

The method of pseudopotentials (IIarrison [16]) is well known for furnishing a reasonably accurate description of the properties of liquid metals. Ilowever, the calculations of the resistivity of liquid metals are beset by difficulties encountered in the measurement of liquid structure factors and in the evaluation of electron-ion interaction potentials, $V(r)$.

An exact formulation of the matrix element for the electron scattering from a state $k$ to $k+q$ is a formidable task. Using Ziman's formula (Shimoji [17]) one can write the resistivity of liquid metals $\left(\rho_{\mathrm{L}}\right)$ as

$$
\rho_{\mathrm{L}}=\frac{12 \pi m^{* 2} \Omega_{0}}{e^{2} \hbar^{3} k_{\mathrm{F}}^{2}} \int_{0}^{2 k_{\mathrm{F}}}|\langle k+q|V| k\rangle|^{2} a(q)\left(\frac{q}{2 k_{\mathrm{F}}}\right)^{3} \mathrm{~d}\left(\frac{q}{2 k_{\mathrm{F}}}\right),
$$

where $m^{*}$ is the effective mass of the conduction electrons, $\Omega_{0}$ is the ionic volume, $q$ is the scattering vector, $k_{F}$ is the Fermi momentum and $a(q)$ is the structure factor. Equation (1) can also be written as

$$
\rho_{\mathrm{L}}=\frac{12 \pi m^{* 2} \Omega_{0}}{e^{2} \hbar^{3} k_{\mathrm{F}}^{2}} \int_{0}^{1} a(q)|V(x)|^{2} x^{3} \mathrm{~d} x
$$

where $x=q / 2 k_{\mathrm{F}}$. In order to calculate the form factor, we choose an Ashcroft empty core pseudopotential (Ashcroft [18])

$$
V^{0}(r)= \begin{cases}0 & r<r_{\mathrm{c}}, \\ -\frac{Z e^{2}}{r} & r \geq r_{\mathrm{c}}\end{cases}
$$

where $r_{c}$ is the empty core radius and $Z$ is the valency.

The Fourier transform of Eq. (3) in momentum space gives

$$
V^{0}(q)=-\frac{4 \pi Z e^{2}}{\Omega_{0} q^{2}} \cos \left(q r_{\mathrm{c}}\right)
$$

$r_{\mathrm{c}}$ can be calculated by minimizing the binding energy with respect to the radius $r_{\mathrm{s}}$ of the spherical space that each electron occupies which leads to a formula (Azez and Kachhava [15])

$$
r_{\mathrm{c}}^{2}=\frac{r_{\mathrm{s}}^{3}}{9}\left[0.031+\frac{1.8 Z^{2 / 3}}{r_{\mathrm{s}}}+\frac{0.916}{r_{\mathrm{s}}}-\frac{4.42}{r_{\mathrm{s}}^{2}}\right] \text {. }
$$

The structure factor $a(q)$ can be calculated theoretically by using a hard sphere model, where a general relation between $a(q)$ and the Fourier transform of the direct correlation functions, $c(q)$, exists (Shimoji [17]),

$$
a(q)=[1-n c(q)]^{-1},
$$


where $n$ is the number of ions per unit volume $\left(n=1 / \Omega_{2}\right)$ and

$$
\begin{gathered}
n c(q)=\frac{-24 \eta}{(1-\eta)^{4} q^{6} \sigma^{6}}\left\{(1+2 \eta)^{2} q^{3} \sigma^{3}[\sin (q \sigma)-q \sigma \cos (q \sigma)]\right. \\
-6 \eta\left(1+\frac{1}{2} \eta\right)^{2} q^{2} \sigma^{2}\left[2 q \sigma \sin (q \sigma)-\left(q^{2} \sigma^{2}-2\right) \cos (q \sigma)-2\right] \\
\left.+\frac{1}{2} \eta(1+2 \eta)^{2}\left[\left(4 q^{3} \sigma^{3}-24 q \sigma\right) \sin (q \sigma)-\left(q^{4} \sigma^{4}-12 q^{2} \sigma^{2}+24\right) \cos (q \sigma)+24\right]\right\},
\end{gathered}
$$

where $\eta=\pi \sigma^{3} /\left(6 \Omega_{0}\right)$ is the packing fraction, $\sigma$ is known as a hard sphere diameter. We have adopted $\eta=0.46$ for all liquid metals (Shimoji [17]).

By substituting Eq. (7) into Eq. (6), the expression for the hard sphere structure factor $a(q)$ can be determined. We have used this expression to calculate the structure factor for liquid metals $\mathrm{K}, \mathrm{Al}, \mathrm{Sn}$ and $\mathrm{Bi}$.

In order to calculate $\rho_{\mathrm{L}}$ from Eq. (7), a screened form factor is used. It is obtained by dividing $V^{0}(q)$ by a modified Ilartree dielectric function, $\epsilon^{*}(q)$, i.e.,

$$
V(q)=\frac{V^{0}(q)}{\epsilon^{*}(q)}
$$

with

$$
\epsilon^{*}(q)=1+\frac{\left[4 k_{\mathrm{F}} /\left(\pi q^{2}\right)\right] \epsilon(q)}{1-\left[4 k_{\mathrm{F}} /\left(\pi q^{2}\right)\right] \epsilon(q) f(q)}
$$

and

$$
\epsilon(q)=\frac{1}{2}+\frac{\left(x^{2}-1\right)}{4 x} \ln \left|\frac{1-x}{1+x}\right|,
$$

$f(q)$ describes the exchange-correlation effects of the electrons.

The resistivity of liquid metals depends upon the nature of the dielectric screening of ions by conduction electrons. We consider the screening functions due to IIartree (II), IIarrison [16], Geldart and Vosko (GV) [19], Hubbard (IIB) [20], Overhauser (OII) [21], random phase approximation (RPA) and self-consistent screening (SCS) [22].

The IIubbard (IIB), Geldart and Vosko (GV), SCS and Overhauser (OH) screening functions are given respectively by [23]:

$$
\begin{aligned}
& f_{\mathrm{HB}}(q)=\frac{0.5 q^{2}}{q^{2}+k_{\mathrm{F}}^{2}+(4 / \pi) m^{*} k_{\mathrm{F}}} \\
& f_{\mathrm{GV}}(q)=\frac{0.5 q^{2}}{q^{2}+\left[2 /\left(1+0.026 m^{*} r_{\mathrm{s}}\right)\right] k_{\mathrm{F}}^{2}} \\
& f_{\mathrm{SCS}}(q)=A\left\{1-\exp \left[B\left(\frac{q}{k_{\mathrm{F}}}\right)^{2}\right]\right\}
\end{aligned}
$$

and

$$
f_{\mathrm{OII}}(q)=\frac{0.275\left(q / k_{\mathrm{F}}\right)^{2}}{\left[1+2.5\left(q / k_{\mathrm{F}}\right)^{2}+0.09375\left(q / k_{\mathrm{F}}\right)^{4}\right]^{1 / 2}} .
$$

The constants $A$ and $B$ appearing in Eq. (13) can be determined by curve fitting as was done by Singwi et al. [22]. 


\section{Results and discussion}

We calculated the resistivity for $\mathrm{K}, \mathrm{Al}, \mathrm{Sn}$ and $\mathrm{Bi}$ by using Ziman's formula considering both the experimental structure factor $a(q)[24,25]$ and the theoretical value based on a hard sphere model as obtained here. The results with different screening functions are tabulated in Table I and II (also the values of $\Omega_{0}, k_{\mathrm{F}}$, $m^{*}$ and $\sigma$ are tabulated in Table III). The calculated results are in very good agreement with experimental observation. It is quite apparent that the calculation of resistivity depends very much upon the choice of screening functions and the structure factors.

TABLE I

Resistivity of liquid metals $(\mu \Omega \mathrm{cm}$ ) with diferent screening functions due to Hartree (H), random phase approximation (RPA) and Hubbard (HB). We use experimental values of structure factor $a(q)$ in a.u. and calculated $a(q)$ from Eqs. (6) and (7).

\begin{tabular}{|c|c|c|c|c|c|c|c|c|}
\hline Dielec. & \multicolumn{2}{|c|}{ Hartree } & \multicolumn{2}{|c|}{ RPA } & \multicolumn{2}{|c|}{$\mathrm{HB}$} & \multirow{3}{*}{$\begin{array}{l}\text { Exp. } \\
\text { values } \\
\text { of } \\
\text { resist. }\end{array}$} & \multirow{3}{*}{$\begin{array}{c}\text { Melting } \\
\text { point } \\
{\left[{ }^{\circ} \mathrm{C}\right]}\end{array}$} \\
\hline Liquid & $a(q)$ & $a(q)$ & $a(q)$ & $a(q)$ & $a(q)$ & $a(q)$ & & \\
\hline metals & Exp. & Cal. & Exp. & Cal. & Exp. & Cal. & & \\
\hline $\mathrm{K}$ & $9.82^{a}$ & 11.03 & $9.89^{a}$ & 11.13 & $8.63^{a}$ & 9.66 & $12.97^{b}$ & $63.50^{b}$ \\
\hline $\mathrm{Al}$ & $27.40^{c}$ & 26.92 & $27.20^{c}$ & 26.89 & $26.20^{c}$ & 26.00 & $24.20^{b}$ & $659^{b}$ \\
\hline Sn & $51.88^{c}$ & 50.19 & $55.92^{c}$ & 53.67 & $47.32^{c}$ & 27.00 & $48.00^{b}$ & $232^{b}$ \\
\hline $\mathrm{Bi}$ & $136.05^{c}$ & 137.88 & $142.05^{c}$ & 144.61 & $130.89^{c}$ & 133.55 & $128.10^{b}$ & $271.30^{b}$ \\
\hline
\end{tabular}

${ }^{a}$ Ref. [24], ${ }^{b}$ Ref. [17], ${ }^{c}$ Ref. [25].

\section{TABLE II}

Resistivity of liquid metals $(\mu \Omega \mathrm{cm})$ with diferent screening functions due to Geldart and Vosko (GV), self-consistent screening (SCS) and Overhauser (OII). We use experimental values of structure factor $a(q)$ (a.u.) and calculated $a(q)$ (a.u.) from Eqs. (6) and (7).

\begin{tabular}{|c|c|c|c|c|c|c|c|c|}
\hline Dielec. & \multicolumn{2}{|c|}{ GV } & \multicolumn{2}{|c|}{$\overline{S C S}$} & \multicolumn{2}{|c|}{$\mathrm{OH}$} & \multirow{3}{*}{$\begin{array}{c}\text { Exp. } \\
\text { values } \\
\text { of } \\
\text { resist. }\end{array}$} & \multirow{3}{*}{$\begin{array}{c}\text { Melting } \\
\text { point } \\
{\left[{ }^{\circ} \mathrm{C}\right]}\end{array}$} \\
\hline$\overline{\text { Liquid }}$ & $a(q)$ & $a(q)$ & $a(q)$ & $a(q)$ & $a(q)$ & $a(q)$ & & \\
\hline metals & Exp. & Cal. & Exp. & Cal. & Exp. & Cal. & & \\
\hline $\mathrm{K}$ & $8.14^{a}$ & 9.53 & $7.35^{a}$ & 7.99 & $8.37^{a}$ & 9.44 & $12.97^{b}$ & $63.50^{b}$ \\
\hline $\mathrm{Al}$ & $25.97^{c}$ & 25.01 & $24.92^{c}$ & 24.66 & $26.13^{c}$ & 25.41 & $24.20^{6}$ & $659^{b}$ \\
\hline Sn & $44.99^{c}$ & 43.33 & $41.04^{c}$ & 40.41 & $46.21^{c}$ & 45.86 & $48.00^{6}$ & $232^{b}$ \\
\hline $\mathrm{Bi}$ & $128.28^{c}$ & 130.59 & $121.16^{c}$ & 125.10 & $129.73^{c}$ & 130.99 & $128.10^{b}$ & $271.30^{b}$ \\
\hline
\end{tabular}

${ }^{a}$ Ref. [24], ${ }^{b}$ Ref. [17], ${ }^{c}$ Ref. [25].

The impact of the screening function on $a(q)$ is different for different metals and may cause variations up to $20 \%$. It appears that the same screening function 


\section{TABLE III}

The input experimental data: the valency $Z$, Fermi wave number $k_{\mathrm{F}}$, and the atomic volume $\Omega_{0}$, were taken from Shimoji [17], $m^{*}$, the effective electron mass, was taken from Heine et al. [26] and the hard sphere diameter $(\sigma)$ was calculated.

\begin{tabular}{c|c|c|c|c|c}
\hline \hline $\begin{array}{c}\text { Liquid } \\
\text { metals }\end{array}$ & Valency & $\begin{array}{c}\Omega_{0} \\
\text { [a.u.] }\end{array}$ & $\begin{array}{c}k_{\mathrm{F}} \\
{[\text { [.u. }}\end{array}$ & $\begin{array}{c}m^{*} \\
\text { [a.u.] }\end{array}$ & $\begin{array}{c}\sigma_{\text {cal. }} \\
\text { [a.u.] }\end{array}$ \\
\hline $\mathrm{K}$ & 1 & $533.1^{a}$ & $0.381^{a}$ & $0.990^{d}$ & 7.76 \\
$\mathrm{Al}$ & 3 & $127.9^{a}$ & $0.885^{a}$ & $0.920^{d}$ & 4.82 \\
$\mathrm{Sn}$ & 4 & $190.8^{a}$ & $0.850^{a}$ & $0.920^{d}$ & 5.51 \\
$\mathrm{Bi}$ & 5 & $233.4^{a}$ & $0.858^{a}$ & $0.930^{d}$ & 5.89 \\
\hline
\end{tabular}

${ }^{a}$ Ref. [17], ${ }^{d}$ Ref. [26].

does not work uniformly well for all metals. For example, the results obtained for $\mathrm{K}$ with IIartree and RPA are closer to experiment than those for other screening functions. For Al, however, SCS screening is most suitable. HB screening function works very well for $\mathrm{Sn}$ while GV and OII screening functions are able to yield good results for $\mathrm{Bi}$. It is interesting to observe that IIartree or RPA yield considerably satisfactory results for simple metals such as $\mathrm{K}$ but fail to account for the screening effect in Bi. This is expected in view of complexities associated with Bi which is known to have a fluctuating valency. There might exist a Brillouin zone in $\mathrm{Bi}$ which may contain five electrons per atom, and these high valency electrons may overlap. Therefore, the exchange and correlation effects are more subtle in $\mathrm{Bi}$.

Both, theoretically calculated and experimentally measured structure factors are found to give good results for $\mathrm{Al}, \mathrm{Sn}$ and $\mathrm{Bi}$. The difference is comparatively bigger in the case of $K$. This is because the structure factors affect the evaluation of the integral in Ziman's formula whose limit of integration is given by $q=2 k_{\mathrm{F}}$. For monovalent liquid metals, like $K$, the limit of integration lies just to the left of the main peak of $a(q)$. That is to say that the hard sphere $a(q)$ calculated for $\mathrm{K}$ differs from that observed from diffraction experiments for low $q$-values. For higher valent metals, like $\mathrm{Al}, \mathrm{Sn}$ and $\mathrm{Bi}$, the limit of integration extends further to the right of the main peak. Therefore, the difference in theoretical and the experimental values of $a(q)$ at low and high momentum transfers is averaged in the calculation of resistivity.

\section{Acknowledgmont}

We would like to thank Prof. R.N. Singh (Sultan Qaboos University, Oman), Prof. C.A. Hogarth (Brunel University, Uxbridge, U.K.), and Prof. William Oliver (University of Arkansas, U.S.A.) for their valuable discussions and suggestions. 


\section{References}

[1] N.H. March, Can. J. Phys. 65, 219 (1987).

[2] W.H. Young, Can. J. Phys. 65, 241 (1987).

[3] D. Stroud, N.W. Ashcroft, Phys. Rev. B 5, 371 (1972).

[4] I.H. Umar, A. Meyer, M. Watabe, W.II. Young, J. Phys. F 4, 1691 (1974).

[5] J. Hafner, Phys. Rev. A 16, 351 (1977).

[6] R.N. Singh, J. Phys. F 10, 1411 (1980).

[7] C. Regnault, J. Phys. F 16, 295 (1986).

[8] D.K. Pandey, R.N. Singh, P.L. Srivastava, J. Phys. Chem. Liq. 18, 151 (1988).

[9] H. Jones, J. Chem. Pliys. 55, 2640 (1971).

[10] N.W. Ashcroft, D. Langreth, Phys. Rev. 150, 500 (1967).

[11] J.D. Weeks, D. Chandler, J. Andersen, J. Chem. Phys. 54, 5237 (1971).

[12] J.P. Hansen, Phys. Rev. A 8, 3096 (1973).

[13] K.A. Azez, M.M. Gupta, C.M. Kachliava, Acta Phys. Hung. 68, 41 (1990).

[14] K.A. Azez, P.C. Agarwal, C.M. Kachliava, Acla Phys. Ifung. 70, 15 (1991).

[15] K.A. Azez, C.M. Kachhava, Ind. J. Pure Appl. Phys. 20, 811 (1991).

[16] W.A. Harrison, Pseudopotentials in the Theory of Metals, W.A. Benjamin Inc., New York 1966.

[17] M. Shimoji, Liquid Metals, Academic Press, London 1977.

[18] N.W. Ashcroft, Phys. Lett. 23, 48 (1966).

[19] D.J.W. Geldart, S.H. Vosko, Can. J. Phys. 44, 2137 (1966).

[20] K.N. Falicov, V. Heine, Adv. Phys. 10, 57 (1961).

[21] A.W. Overhauser, Phys. Rev. B 3, 1888 (1971).

[22] K.S. Singwi, A. Sjolander, M.P. Tosi, R.II. Lond, Phys. Rev. B 1, 1044 (1970).

[23] C.M. Kachhava, Solid State Commun. 11, 1439 (1972).

[24] A.J. Greenfield, J. Wellendorf, Phys. Rev. A 4, 1607 (1971).

[25] Y. Waseda, K. Suzuki, Phys. Status Solidi B 49, 339 (1972).

[26] V. Heine, D. Weaire, Solid State Phys. 24, 67 (1970). 